\title{
Bacterial Polysaccharides - Potential Candidate for Vaccine Development
} Pillai TG ${ }^{1^{*}}$ and Mini $\mathbf{M}^{2}$

${ }^{1}$ Department of Forest Pathology, Kerala Forest Pathology, Peechi, Thrissur, India

${ }^{2}$ Department of Veterinary Microbiology, College of Veterinary and Animal Sciences, Mannuthy, Thrissur, India

${ }^{*}$ Corresponding author: Pillai TG, Department of Forest Pathology, Kerala Forest Pathology, Peechi, Thrissur, India, Tel: 91-487-2690322; E-mail: thulasigpilla@yahoo.co.in

Rec date: Oct 14, 2015; Acc date: Mar 21, 2016; Pub date: Mar 24, 2016

Copyright: (c) 2016 Pillai TG, This is an open-access article distributed under the terms of the Creative Commons Attribution License, which permits unrestricted use, distribution, and reproduction in any medium, provided the original author and source are credited.

\begin{abstract}
Capsular polysaccharides are important surface components of bacteria. These are virulence factors. Pasteurella multocida (DP1) was isolated from ducklings. The polysaccharide components were isolated from $P$. multocida (DP1) strain. The structural elucidations were done by Infra-Red (IR) and nuclear magnetic resonance (NMR) spectrum. The polysaccharides are potential candidates for development of vaccines.
\end{abstract}

Keywords: P. multocida; DP1; Polysaccharides; Vaccines; IR; NMR

\section{Introduction}

P. multocida is the etiological agent of fowl cholera, an extremely contagious disease of wild and domestic birds that inflicts serious economic losses on the poultry industry [1]. Recognised by Louis Pasteur as one of the important infectious diseases [2]. The etiological agent of fowl cholera in avian species like chicken, turkey and waterfowls. It causes septicemia in birds and rapidly fatal. Carrier birds play a major role in the transmission of fowl cholera. Based on capsule antigens, they are 5 serogroups - A, B, D, E and F. Fowl cholera is generally caused by A:1, A:3 or A4 strains. In this study we isolated the capsular PS and the structure was studied.

\section{Materials and Methods}

The cultures of DP1 were maintained in brain heart infusion agar. For production of capsular polysaccharides the cultures were grown in Brain heart infusion broth with chitin flakes, with occasional shaking. Chitin flakes enhances the production of capsule. The cells were removed by centrifugation at $6000 \mathrm{rpm}$. The supernatant containing the polymeric material was concentrated and precipitated with 2.5 volumes of ethanol. The resulting pellet was dissolved in water and treated with Sevag's reagent $\left[\mathrm{CHCl}_{3}: \mathrm{CH}_{3} \mathrm{OH}\right.$ (3:1)] proportion to remove the protein. The solution containing the capsular material was treated with equal volume of cetyl trimethyl ammonium hydroxide and refrigerated for 12 hours. The supernatant obtained was treated with 2.5 times of ethanol and refrigerated for 48 hours. The precipitate is centrifuged and dried to obtain light brown powder, the capsular polysaccharide. The compound was completely soluble in DDW and the dissolved solution is used for Infra red (IR) spectroscopy and Nuclear Magnetic Resonance (NMR) spectroscopy. The IR and NMR spectroscopy was done at IIT, Bombay, Mumbai, India.

\section{Sugar Analysis}

Sugar analysis was done by Anthrone test [3].

\section{Results}

The sugar analysis confirmed that the compound was carbohydrate. In the IR spectrum, absorption peaks were observed at $34.51 \mathrm{~cm}^{-1}$, $2926 \mathrm{~cm}^{-1}$ and $1642 \mathrm{~cm}^{-1}$ indicating $\mathrm{OH}$ stretching, a C-H bond and a $\mathrm{C}=\mathrm{O}$ bond respectively [3]. The peak at $920 \mathrm{~cm}^{-1}$ corresponds to $\beta$ $(1,6)$ linkage [4] $\alpha$-D specific peaks for glucan at $850 \mathrm{~cm}^{-1}$ were not found in DP-1 PS spectrum. NMR analysis showed that, $\mathrm{H}$ chemical shift of anomeric region was observed around $4 \sim 6 \mathrm{ppm}$ [5]. Anomeric signals for $\beta-1,-6$ linkage appear at $4.5 \sim 4.6 \mathrm{ppm}$ [6]. The absence of peak in the range $0.5 \sim 3.0 \mathrm{ppm}$ implied that DP1 polysaccharide is pure glucan (Figures 1 and 2).

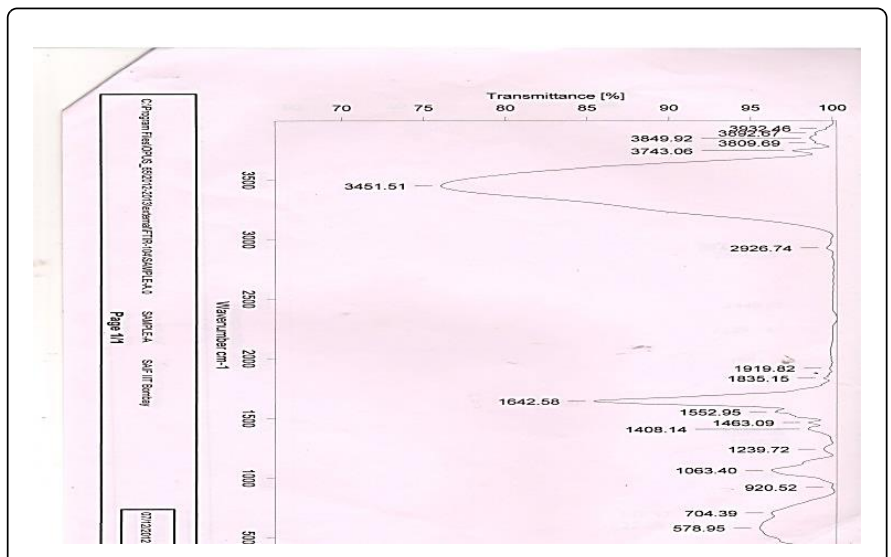

Figure 1: IR analysis. 
Citation: Pillai TG, Mini M (2016) Bacterial Polysaccharides - Potential Candidate for Vaccine Development. J Med Microb Diagn 5: 224. doi: 10.4172/2161-0703.1000224

Page 2 of 2

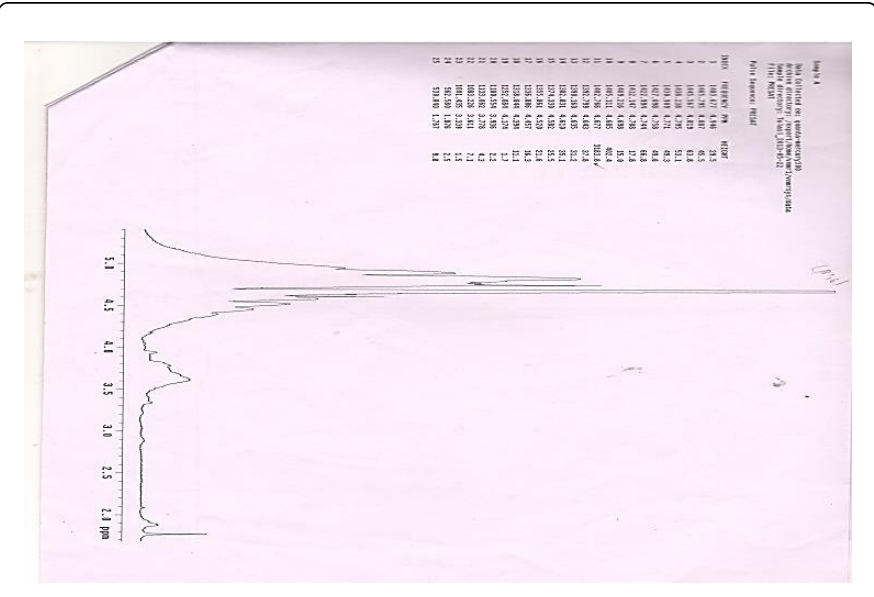

Figure 2: NMR analysis.

\section{Discussion}

Bacteria possess variety of mechanisms to host defenses and capsule is one of the virulence factors. The potential roles of capsular Pasteurella multocida during life cycle include avoiding phagocytosis, defeating complement, modulating host responses of physiology and resisting desiccation [7,8]. Polysaccharides have lot of applications now-a-days. Considering Pasteurella multocida as antigens, immunity against these antigens can confer protection against disease. Pasteurella multocida was the target of several investigators in 1920s and 1930s. Development of vaccines based on capsular polysaccharides is an effective way to fight against disease. From the present study, we arrive at the conclusion that the Pasteurella multocida obtained from DP1 is $\beta$ (1-6) glucan. The antigenic variation possessed by carbohydrate antigens is a limiting factor for vaccine production. Pasteurella multocida utilisation in vaccine production has been partially successful. Further studies will reveal the potential of using DP1-PS for vaccine production.

\section{References}

1. Harper M, Boyce JD, Adler B (2006) "PM pathogenesis: 125 years after Pasteur". FEMS Microbiol Lett 265: 1-10.

2. Carpenter TE, Snipes KP, Wallis D, Mc Capes R (1988) Epidemiology and Financial effect of foul cholera in turkeys: a retrospective analysis. Avian Dis 32 : 16-23.

3. Jung HK, Hong JH, Park SC, Park BK, Nani DH, et al. (2007) Production and physiochemical characterisation of $\beta$-glucan produced by Paenibacillus polymyxa JB 115. Biotechnology \& Bioprocess Engineering 12: 713-719.

4. Sandula J, Kogan G, Kacurakova M, Machova E (1999) Microbial (1-73) $\beta$-D-glucans, their preparation, physiochemical characterisation and immuno-modulatory activity. Carbohydr Polym 38: 247-253.

5. Gonzaga MLC, Ricardo NMPS, Heatley F, de A Soares S (2005) Carbohyd Poly 60: 43-49.

6. Sugawara T, Carb Res 339: 2255-2265.

7. Roberts IS (1996) Annu Rev Microbiol 50: 285-315.

8. Yemn EW, Wills AJ (1954) The estimation of carbohydrate in plant extract by anthrone. Biochem J 57: 508-514. 